\title{
Optimal Wake-up Mechanism for Single Base Station with Sleep Mode
}

\author{
Xueying Guo, Sheng Zhou, Zhisheng Niu \\ Tsinghua National Laboratory for Information Science and Technology \\ Dept. of Electronic Engineering, Tsinghua Univ. \\ Beijing, 100084, P.R. China \\ guo-xy11@mails.tsinghua.edu.cn, $\{$ sheng.zhou, niuzhs $\} @$ tsinghua.edu.cn
}

\author{
P.R. Kumar \\ Computer Engineering and Systems Group \\ Department of ECE, Texas A\&M Univ. \\ College Station, TX 77843, USA \\ prk@tamu.edu
}

\begin{abstract}
Base station (BS) sleeping is an effecting way to improve the energy-efficiency of cellular networks. Considering BS sleep mode operation under different scenarios, we focus on three wake-up policies: single vacation (SV) policy, multiple vacation (MV) policy and $N$ policy. A hysteresis time is also considered to avoid frequent BS mode-changing operation. By modeling the systems as M/G/1 vacation queues, we derive two performance measures of interest, expected system response time and energy consumption per bit. The impacts of sleep mode operation parameters and setup time are also studied. In order to determine the optimal parameter settings, which allows for a flexible tradeoff between energy-efficiency and mean delay, a two-step optimization method is proposed. We numerically analyze and investigate the energy-delay tradeoff for different policies, and find that both the MV policy and the $N$ policy have better performance than the SV policy if the sniffing cost is not considered. However, these advantages diminish when the sniffing cost increases.
\end{abstract}

\section{INTRODUCTION}

The ever increasing demand for ubiquitous information access and broadband multimedia service in wireless networks has triggered vast expansion of network infrastructures, resulting in dramatically increased energy consumption. Among the infrastructures, base stations (BS) contribute to 60-80 percent of the cellular network energy consumption [1]. As a result, improving BS energy-efficiency is important for the green evolution of wireless networks.

BS sleeping is an effective way to improve BS energyefficiency while maintaining acceptable quality of service (QoS) [1], [2], [3], [4]. To save energy, the BS or part of it can be switched off when the traffic load is low, but the QoS then deteriorates accordingly. In this paper, we treat the QoS problem accompanying with BS sleep mode from a different perspective: we investigate how much energy can be traded off for a certain amount of delay. Solving this tradeoff problem should demonstrate the cost of improving the energy-efficiency through BS sleep mode, and at the same time throw light upon the pricing policy of operators.

There are some previous studies: in [5] it is shown that energy-delay tradeoff does exist in fading channels; in [6] the energy-delay tradeoff by dynamic on/off operation of BSs and user associations is explored; in [7] power adaptation and sleep control are combined to evaluate the relationship. However, these previous works either omit the design of a specific wakeup policy or ignore practical constraints such as setup time and sniffing cost.

BS mode-changing operation incurs an extra energy and performance penalty. To avoid frequent BS mode-changing operation, we introduce a hysteresis time in the BS sleep policy: when the system becomes empty, a timer is activated, and the BS is switched off only if the system remains empty at its expiration. The sniffing cost, the additional energy for detecting whether there are waiting customers, should also be considered, which is closely related to the wake-up policy. Specifically, we consider three wake-up policies as follows:

- In the single vacation (SV) policy, the BS wakes up after a specified period of time. This is applicable when the BS cannot detect whether there are waiting customers in sleep mode.

- In the multiple vacation (MV) policy, the BS takes a vacation when sleep mode begins. After a vacation time, it takes another vacation if the system is still empty, or otherwise wakes up. This is applicable when the BS can be partly switched on to detect whether there are waiting customers.

- In the $N$ policy, the BS remains in sleep mode until there are $N$ customers waiting in the system. This is applicable when the BS keeps counting coming costumers in sleep mode by itself or with the help of other network equipments.

This work is a generalization of [8], which only considers the $N$ policy and omits sniffing cost.

We model the BS with sleep mode as an M/G/1 vacation queue with setup and close-down times. For each wake-up policy, we exploit the impacts of operation parameters and setup time. In addition, we propose a two-step optimization method to achieve optimal energy-delay tradeoff and to choose optimal operation parameters.

This paper is organized as follows. In section II, we describe our system model and notations. In section III, we analyze the queueing system. In section IV, we further exploits the impacts of important parameters. Section V deals with the optimization method, concluding in section VI. 


\section{SySTEM MODEL}

\section{A. The Queueing Model}

A single BS with sleep mode is modeled as an M/G/1 vacation queue with setup and close-down times by considering the whole BS as a single server. Requests arrive at the BS as a Poisson process of rate $\lambda$. The service times are assumed to be generally distributed and independent and identically distributed with mean $\mu^{-1}$ and coefficient of variation $c_{b}^{2}$.

The hysteresis time is modeled as a close-down time. In the queue with vacations, when the system becomes empty, the server enters a close-down time period. If no customers arrive during the close-down time, then the server enters sleep mode; otherwise, the server retains in active mode. The length of close-down time, denoted by $D$, is assumed to be generally distributed. The Laplace-Stieltjes transform (LST) of $D$ is denoted by $D^{*}(s)$.

The ending time of the sleep mode depends on the wake-up policy:

- For the SV policy, the server returns to active mode after a specified vacation time. The length of the vacation time, denoted by $V$, is assumed to be generally distributed with coefficient of variation $c_{v}^{2}$ and $\operatorname{LST} V^{*}(s)$.

- For the MV policy, the server takes a vacation when sleep mode begins; after a vacation time, it takes another vacation if the queue is still empty, or otherwise returns to active mode. The length of each single vacation, denoted by $V$, is assumed to be identically distributed with coefficient of variation $c_{v}^{2}$ and $\operatorname{LST} V^{*}(s)$.

- For the $N$ policy, the server remains in sleep mode until there are $N$ customers waiting in the system.

When transitioning into active mode, the server needs a setup time. The length of the setup time, denoted by $S$, is assumed to be generally distributed with coefficient of variation $c_{s}^{2}$, and LST $S^{*}(s)$.

Fig. 1 shows how the server operates in the SV policy. It should be noted that the SV policy here is different from the single vacation model with setup and close-down times in some existing literature [9], [10], [11], [12], since in our case the server goes to the setup state immediately after the single vacation regardless of whether there are waiting customers.

\section{B. The Energy Consumption Model}

The operation of the BS can be divided into several time periods: the busy period, when the BS serves the customers; the close-down period; the sleep period; the setup period, and the idle period, when the BS is in active mode with no customers. It should be noted that the idle period only exists in the SV policy.

Let us denote the BS power consumption during different periods by $P_{\mathrm{BS}}, P_{\mathrm{CD}}, P_{\mathrm{SL}}, P_{\mathrm{ST}}$, and $P_{\mathrm{ID}}$ respectively. The time ratios of the different periods, where the time ratio means the percent of time that the BS spends in a specific operation time period as a fraction of the total time under consideration, are denoted by $\eta_{\mathrm{BS}}, \eta_{\mathrm{CD}}, \eta_{\mathrm{SL}}, \eta_{\mathrm{ST}}, \eta_{\mathrm{ID}}$ respectively.

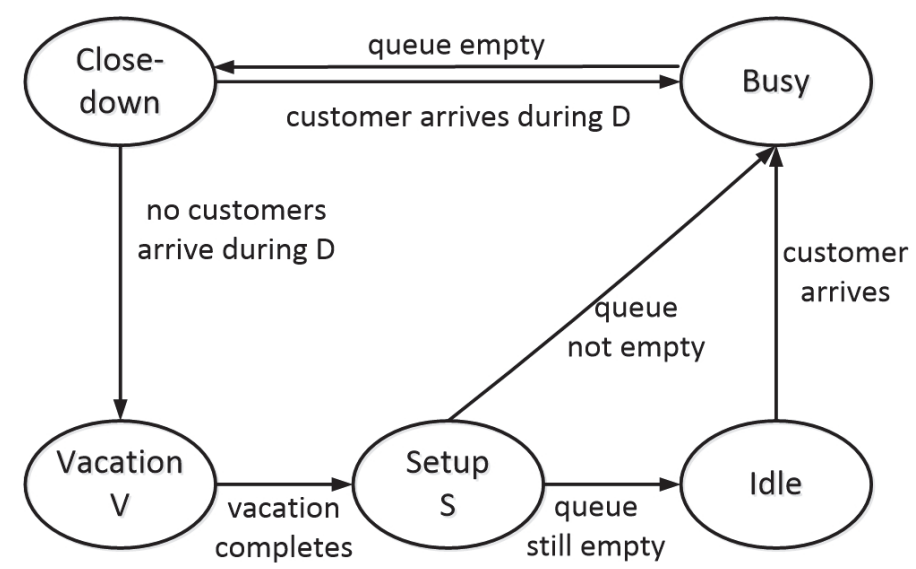

Fig. 1. State transition diagram of M/G/1 vacation queue with setup and close-down times under the SV policy

We will employ the linear approximation of the BS power as in [13]:

$$
P= \begin{cases}P_{0}+\Delta_{p} P_{\mathrm{t}}, & \text { in active mode } \\ P_{\mathrm{SL}}, & \text { in sleep mode }\end{cases}
$$

where $P_{0}$ and $P_{\mathrm{t}}$ represent the static power consumption in active mode and the transmission power, respectively. $\Delta_{p}$ is the slope of the load dependent power consumption. It can be easily inferred that $P_{\mathrm{BS}}=P_{0}+\Delta_{p} P_{\mathrm{t}}$ and $P_{\mathrm{CD}}=P_{\mathrm{ID}}=P_{0}$.

As a result, the energy consumption per bit, which is the metric of energy-efficiency, is

$$
\begin{aligned}
E_{\mathrm{bit}}= & \frac{1}{\lambda \bar{L}_{\mathrm{data}}}\left[\eta_{\mathrm{BS}} P_{\mathrm{BS}}+\eta_{\mathrm{CD}} P_{\mathrm{CD}}+\eta_{\mathrm{SL}} P_{\mathrm{SL}}\right. \\
& \left.+\eta_{\mathrm{ST}} P_{\mathrm{ST}}+\eta_{\mathrm{ID}} P_{\mathrm{ID}}+P_{\mathrm{cost}}\right]
\end{aligned}
$$

where $\bar{L}_{\text {data }}$ represents the average data length. The sniffing cost $P_{\text {cost }}$ above depends on the wake-up policy as follows:

$$
P_{\text {cost }}= \begin{cases}0 & \text { for the SV policy, } \\ \lambda_{\mathrm{V}} E_{\text {sniff }} & \text { for the MV policy, } \\ \eta_{\mathrm{SL}} P_{\text {sniff }} & \text { for the } N \text { policy, }\end{cases}
$$

where $\lambda_{\mathrm{V}}$ is the rate of vacations, $E_{\text {sniff }}$ represents the energy consumption of each detection for the MV policy, and $P_{\text {sniff }}$ represents the extra power consumption for detecting arriving customers during the sleep period in the $N$ policy.

The service rate $\mu$ during the busy period is closely related to the transmission power $P_{\mathrm{t}}$ :

$$
\mu=\frac{B_{\mathrm{w}}}{\bar{L}_{\text {data }}} \cdot \log _{2}\left(1+\gamma P_{\mathrm{t}}\right), \gamma=\frac{g}{N_{0} B_{\mathrm{w}}},
$$

where $g^{1}, B_{\mathrm{w}}$ and $N_{0}$ denote the channel gain, bandwidth and noise spectral density, respectively.

\footnotetext{
${ }^{1}$ Since our focus is on BS sleep mode operation which does not affect the transmission power, we study the basic case where all users experience a homogeneous channels with gain $\mathrm{g}$.
} 


\section{QUeUeING ANALYSis}

Although the three queueing systems have different wake-up policies, they are all M/G/1 vacation queues with exhaustive service, which means the server only switches off when there are no customers in the system. From [14] [15], by stochastic decomposition, the mean sojourn time of customers can be expressed as

$$
\begin{aligned}
T & =T_{M / G / 1}+T_{\mathrm{v}} \\
& =\left[\frac{1}{\mu}+\frac{\lambda\left(1+c_{b}^{2}\right)}{2 \mu(\mu-\lambda)}\right]+\frac{E\left[K_{\mathrm{v}}^{2}\right]-E\left[K_{\mathrm{v}}\right]}{2 \lambda E\left[K_{\mathrm{v}}\right]},
\end{aligned}
$$

where $K_{\mathrm{v}}$ denotes the number of messages that arrive during a vacation period ${ }^{2}$. It should be noted that in the expression for the mean sojourn time in (4), the term in the square brackets is the mean sojourn time of a standard $\mathrm{M} / \mathrm{G} / 1$ queue, while the last part is the additional delay due to vacations, denoted by $T_{\mathrm{v}}$.

By deriving the Z-transform of $K_{\mathrm{v}}$, we can determine $T_{\mathrm{v}}$ for different wake-up policies:

$$
\begin{aligned}
T_{\mathrm{v}}^{(\mathrm{SV})} & =\frac{\frac{1}{2} \lambda D^{*}(\lambda) E\left[(V+S)^{2}\right]}{D^{*}(\lambda)\left[\lambda E[S+V]+V^{*}(\lambda) S^{*}(\lambda)-1\right]+1} ; \\
T_{\mathrm{v}}^{(\mathrm{MV})} & =\frac{\frac{\lambda D^{*}(\lambda)}{1-V^{*}(\lambda)}\left\{E\left[(V+S)^{2}\right]-V^{*}(\lambda) E\left[S^{2}\right]\right\}}{2\left\{D^{*}(\lambda)\left[\frac{E[V] \lambda}{1-V^{*}(\lambda)}+E[S] \lambda\right]+\left[1-D^{*}(\lambda)\right]\right\}} ; \\
T_{\mathrm{v}}^{(N)} & =\frac{D^{*}(\lambda)\left[N(N-1)+2 N \lambda E[S]+\lambda^{2} E\left[S^{2}\right]\right]}{2 \lambda\left\{D^{*}(\lambda)[N+\lambda E[S]]+\left[1-D^{*}(\lambda)\right]\right\}} .
\end{aligned}
$$

These results are applicable for any "impartial" service discipline ${ }^{3}$.

To obtain the time ratios of the various operation time periods, we derive the expected length of cycle time and the length of each operation period in a single cycle time. Here, the cycle time is the time interval between two successive generation points, which are taken to be the ending times of busy periods.

As a result, we obtain the energy consumption per bit as follows

$$
E_{\text {bit }}=\frac{1}{\lambda \bar{L}_{\text {data }}}\left\{\rho\left[P_{0}+\frac{\Delta_{p}}{\gamma}\left(2^{\frac{\mu \bar{L}_{\text {data }}}{B_{\mathrm{w}}}}-1\right)\right]+(1-\rho) \bar{P}_{\mathrm{v}}\right\},
$$

where $\rho=\lambda / \mu$, and $\bar{P}_{\mathrm{v}}$ is the average power consumption during a vacation period. The quantity $\bar{P}_{\mathrm{v}}$ depends on the wake-up policy.

For the SV policy,

$$
\bar{P}_{\mathrm{v}}^{(\mathrm{SV})}=\frac{A_{1}^{(\mathrm{SV})} P_{0}+A_{2}^{(\mathrm{SV})} P_{\mathrm{SL}}+A_{3}^{(\mathrm{SV})} P_{\mathrm{ST}}}{A_{1}^{(\mathrm{SV})}+A_{2}^{(\mathrm{SV})}+A_{3}^{(\mathrm{SV})}}
$$

\footnotetext{
${ }^{2}$ Referring to [14], vacation period is the time interval between two successive busy periods. It should be noted that vacation period is different from vacation time.

${ }^{3}$ By "impartial" we mean that the order of service is not affected by the service times of waiting messages. It includes first come first service, last come first service, and service in random order.
}

where

$$
\begin{aligned}
A_{1}^{(\mathrm{SV})} & =\frac{1}{\lambda}\left[1-D^{*}(\lambda)\right]+\frac{1}{\lambda} D^{*}(\lambda) V^{*}(\lambda) S^{*}(\lambda), \\
A_{2}^{(\mathrm{SV})} & =D^{*}(\lambda) E[V], \\
A_{3}^{(\mathrm{SV})} & =D^{*}(\lambda) E[S] .
\end{aligned}
$$

For the MV policy

$$
\bar{P}_{\mathrm{v}}^{(\mathrm{MV})}=\frac{A_{1}^{(\mathrm{MV})} P_{0}+A_{2}^{(\mathrm{MV})} P_{\mathrm{SL}}+A_{3}^{(\mathrm{MV})} P_{\mathrm{ST}}+A_{4}^{(\mathrm{MV})}}{A_{1}^{(\mathrm{MV})}+A_{2}^{(\mathrm{MV})}+A_{3}^{(\mathrm{MV})}},
$$

where

$$
\begin{aligned}
A_{1}^{(\mathrm{MV})} & =\left[1-D^{*}(\lambda)\right] \frac{1}{\lambda}, \\
A_{2}^{(\mathrm{MV})} & =D^{*}(\lambda) \frac{E[V]}{1-V^{*}(\lambda)}, \\
A_{3}^{(\mathrm{MV})} & =D^{*}(\lambda) E[S], \\
A_{4}^{(\mathrm{MV})} & =\frac{D^{*}(\lambda) E_{\text {sniff }}}{1-V^{*}(\lambda)} .
\end{aligned}
$$

For the $N$ policy

$$
\bar{P}_{\mathrm{v}}^{(N)}=\frac{A_{1}^{(N)} P_{0}+A_{2}^{(N)}\left(P_{\mathrm{SL}}+P_{\text {sniff }}\right)+A_{3}^{(N)} P_{\mathrm{ST}}}{A_{1}^{(N)}+A_{2}^{(N)}+A_{3}^{(N)}},
$$

where

$$
\begin{aligned}
A_{1}^{(N)} & =\frac{1}{\lambda}\left[1-D^{*}(\lambda)\right], \\
A_{2}^{(N)} & =\frac{N}{\lambda} D^{*}(\lambda), \\
A_{3}^{(N)} & =E[S] D^{*}(\lambda) .
\end{aligned}
$$

It should be noted that for any wake-up policy, the impact of sleep mode operation on energy-efficiency is only reflected in $\bar{P}_{\mathrm{v}}$, while the impact of sleep mode operation on mean delay is only reflected in $T_{\mathrm{v}}$. This will be used in the discussion in the following sections.

\section{IMPACtS OF SLEEP OPERATION PARAMETERS}

\section{A. Single Vacation Policy}

1) Condition for Sleeping Gain: According to (8), when $\bar{P}_{\mathrm{v}}=P_{\mathrm{ID}}$, the energy-efficiency is equivalent to the case without sleep mode. Hence, only when $\bar{P}_{\mathrm{v}}<P_{\mathrm{ID}}$ can the introduction of sleep mode benefit the system; otherwise, the sleep mode will cause extra delay without improving the energy-efficiency. For the SV policy, this condition is equivalent to the following inequality:

$$
E[V]\left(P_{0}-P_{\mathrm{SL}}\right)>E[S]\left(P_{\mathrm{ST}}-P_{0}\right) .
$$

2) Impact of Setup Time: The impact of setup time on energy-efficiency is only manifested through $E[S]$ and $S^{*}(\lambda)$. We consider the case when (12) holds. Then, when $E[S]$ is given, a larger $S^{*}(\lambda)$ leads to a larger $\bar{P}_{\mathrm{v}}^{(\mathrm{SV})}$. By Jensen's Inequality, $S^{*}(\lambda)$ attains its minimum value when $S$ is constant. Hence, when $E[S]$ is given, a constant $S$ maximizes the energy-efficiency. 

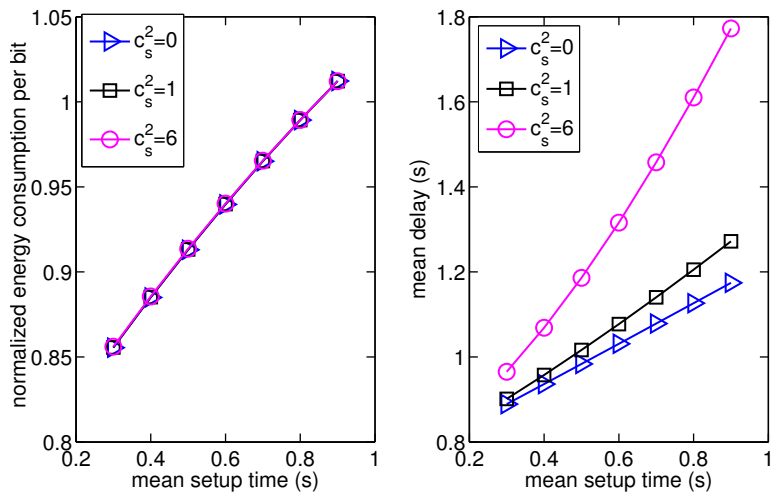

Fig. 2. For the SV policy, the energy consumption per bit (normalized by the case without sleep mode) and mean delay vs. mean setup time for different coefficients of variation of setup time $\left(c_{s}^{2}\right)$ are shown. (for the case of constant vacation time $V$ with $E[V]=2$ s, and exponentially distributed close-down time $D$ with $E[D]=1 \mathrm{~s}$.)

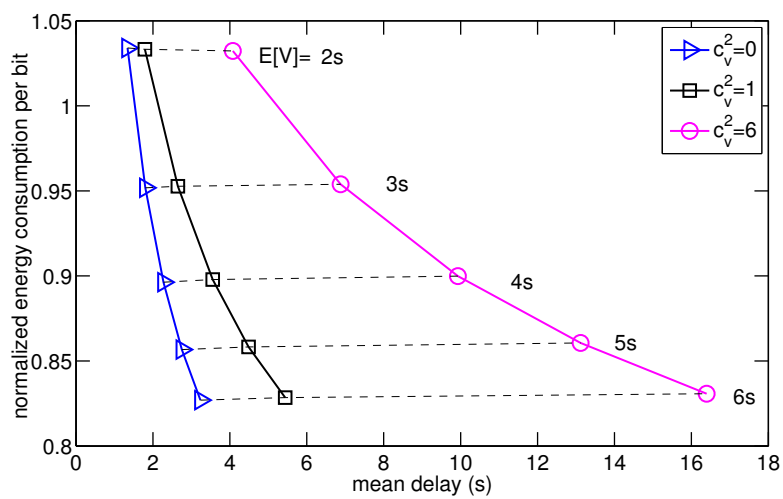

Fig. 3. For the SV policy, the energy consumption per bit (normalized by the case without sleep mode) vs. mean delay by changing mean vacation time $(E[V])$ and for different coefficients of variation of vacation time $\left(c_{v}^{2}\right)$ are shown. (for the case of exponentially distributed setup time $S$ with $E[S]=1$ s, and exponentially distributed close-down time $D$ with $E[D]=1 \mathrm{~s}$. )

A numerical result is shown in Fig. 2. For this case, both smaller mean setup time $(E[S])$ and smaller coefficient of variation of setup time $\left(c_{s}^{2}\right)$ lead to better performance, i.e., better energy-efficiency and smaller mean delay. As a result, when designing BSs, equipment suppliers should make the setup time as short as possible and also reduce the variance of setup time.

3) Impact of Vacation Time: From the numerical results, when (12) holds, we get the best energy-delay relationship when $c_{v}^{2}=0$, which means the vacation time $V$ is constant. An example is shown in Fig. 3.

As a result, to get best performance and to simplify the analysis, we consider the case when vacation time $V$ is constant with value $v$. In this case, when (12) holds, a longer $v$ leads to higher energy-efficiency, but the mean delay is monotonically increase if and only if the following inequality holds:

$$
\begin{array}{r}
D^{*}(\lambda)\left\{2 \lambda(v+E[S])^{2}-\lambda E\left[(v+S)^{2}\right]\left[1-\mathrm{e}^{-\lambda v} S^{*}(\lambda)\right]\right\} \\
+2(v+E[S])\left[1-D^{*}(\lambda)+D^{*}(\lambda) 2 \mathrm{e}^{-\lambda v} S^{*}(\lambda)\right]>0 .
\end{array}
$$

A sufficient condition for (13) is $c_{s}^{2} \leq 1$.

4) Impact of Close-down Time: The impact of closedown time on both energy-efficiency and mean delay is only manifested through $D^{*}(\lambda)$, which is the probability that no customers arrive during the close-down time. When (12) holds, the mean delay increases monotonically as $D^{*}(\lambda)$, but a larger $D^{*}(\lambda)$ also leads to higher energy-efficiency. Hence, for example when the close-down time is exponentially distributed, a larger $E[D]$ leads to smaller mean delay and lower energy-efficiency.

In addition, there is a linear relationship between $\bar{P}_{\mathrm{v}}^{(\mathrm{SV})}$ and $T_{\mathrm{v}}^{(\mathrm{SV})}$ by changing close-down time as follows

$$
\begin{aligned}
\bar{P}_{\mathrm{v}}^{(\mathrm{SV})} & =P_{0}+\frac{2}{E\left[(V+S)^{2}\right]}\left[E[V]\left(P_{\mathrm{SL}}-P_{0}\right)\right. \\
& \left.+E[S]\left(P_{\mathrm{ST}}-P_{0}\right)\right] T_{\mathrm{v}}^{(\mathrm{SV})} .
\end{aligned}
$$

Combining (2), (4) and (14), we can easily deduce that there is a linear energy-delay tradeoff in terms of the changing closedown time.

This linear energy-delay relationship is in consistence with the conclusion in [8], which is different from the energy-delay relationship in terms of changing the service rate in some existing literatures.

\section{B. Multiple Vacation Policy}

1) Condition for Sleeping Gain: As noted earlier, for sleeping gain, we need $\bar{P}_{\mathrm{v}}<P_{\mathrm{ID}}$, which is equivalent to the following inequality:

$$
\frac{E[V]}{1-V^{*}(\lambda)}\left(P_{0}-P_{\mathrm{SL}}\right)>E[S]\left(P_{\mathrm{ST}}-P_{0}\right)+\frac{E_{\mathrm{sniff}}}{1-V^{*}(\lambda)} .
$$

2) Impact of Setup Time: Consider the case when (15) holds. The impact of setup time on energy-efficiency is only manifested through $E[S]$, with a larger $E[S]$ leading to lower energy-efficiency.

The impact of setup time on mean delay is manifested through $E[S]$ and $c_{s}^{2}$. When $E[S]$ is given, the mean delay is a monotonically increasing function of $c_{s}^{2}$. When $c_{s}^{2}$ is given, the mean delay is a monotonically increasing function of $E[S]$ if and only if the following inequality holds:

$$
D^{*}(\lambda) \frac{2 \lambda E[V]^{2}}{1-V^{*}(\lambda)}+2\left(1-D^{*}(\lambda)\right) E[V]-\lambda D^{*}(\lambda) E\left[V^{2}\right] \geq 0 .
$$

Otherwise, there exists an optimal mean setup time which can achieve the minimum mean delay:

$$
E[S]=\sqrt{\frac{F_{s}^{(\mathrm{MV})}}{\left(1+c_{s}^{2}\right)\left(1-V^{*}(\lambda)\right)}}-\frac{E[V]}{1-V^{*}(\lambda)}-\frac{1-D^{*}(\lambda)}{\lambda D^{*}(\lambda)},
$$


where

$$
\begin{aligned}
F_{s}^{(\mathrm{MV})} & =\left[\frac{1-D^{*}(\lambda)}{\lambda D^{*}(\lambda)}\right]^{2}\left(1+c_{s}^{2}\right)\left(1-V^{*}(\lambda)\right)+E\left[V^{2}\right] \\
& -(E[V])^{2}\left(\frac{1-c_{s}^{2}}{1-V^{*}(\lambda)}\right)+\frac{1-D^{*}(\lambda)}{\lambda D^{*}(\lambda)} 2 c_{s}^{2} E[V] .
\end{aligned}
$$

A sufficient condition for (16) is $c_{v}^{2} \leq 1$. As a result, when $c_{v}^{2} \leq 1$, both smaller mean setup time or smaller $c_{s}^{2}$ results in better performance, i.e. better energy-efficiency and smaller mean delay.

3) Impact of Vacation Time: From the numerical results, we get the best energy-delay relationship when $c_{v}^{2}=0$. Hence, we focus on the case when the vacation time is constant with value $v$.

When (15) holds, a larger $v$ results in higher energyefficiency, and the mean delay is a monotonically increasing function of $v$ if and only if the following inequality holds:

$$
\begin{aligned}
& \lambda D^{*}(\lambda) v^{2}+\lambda D^{*}(\lambda)\left(2 E[S]^{2}-E\left[S^{2}\right]\right) F_{2}^{(\mathrm{MV})} \\
+ & \lambda D^{*}(\lambda) E[S] v F_{1}^{(\mathrm{MV})}+\left(1-D^{*}(\lambda)\right) F_{3}^{(\mathrm{MV})} \geq 0,
\end{aligned}
$$

where

$$
\begin{gathered}
F_{1}^{(\mathrm{MV})}=2-\mathrm{e}^{-\lambda v}(2+\lambda v), F_{2}^{(\mathrm{MV})}=1-\mathrm{e}^{-\lambda v}(1+\lambda v), \\
F_{3}^{(\mathrm{MV})}=v F_{1}^{(\mathrm{MV})}-2 E[S] F_{2}^{(\mathrm{MV})} .
\end{gathered}
$$

By exploiting the high-order partial derivatives of $F_{1}^{(\mathrm{MV})}$, $F_{2}^{(\mathrm{MV})}$ and $F_{3}^{(\mathrm{MV})}$ with respect to $v$, we prove that a sufficient condition for (17) is $\lambda \leq 1 / E[S]$ and $c_{s}^{2} \leq 1$.

4) Impact of Close-down Time: When (15) holds, similar to the case of the SV policy, by changing close-down time, a linear tradeoff between energy-efficiency and mean delay exists since

$$
\begin{aligned}
\bar{P}_{\mathrm{v}}^{(\mathrm{MV})}= & P_{0}+\frac{2 T_{\mathrm{v}}^{(\mathrm{MV})}\left(1-V^{*}(\lambda)\right)}{E\left[(V+S)^{2}\right]-V^{*}(\lambda) E\left[S^{2}\right]} \\
& \cdot\left[\frac{E[V]\left(P_{\mathrm{SL}}-P_{0}\right)+E_{\mathrm{sniff}}}{1-V^{*}(\lambda)}+E[S]\left(P_{\mathrm{ST}}-P_{0}\right)\right] .
\end{aligned}
$$

A numerical result is shown in Fig. 4.

\section{C. $N$ Policy}

1) Condition for Sleeping Gain: As noted earlier, for sleeping gain, we need $\bar{P}_{\mathrm{v}}<P_{\mathrm{ID}}$, which is equivalent to the following inequality:

$$
N\left(P_{0}-P_{\mathrm{SL}}-P_{\text {sniff }}\right)>E[S] \lambda\left(P_{\mathrm{ST}}-P_{0}\right) .
$$

2) Impact of Setup Time: We consider the case when (19) holds. The impact of setup time on energy-efficiency is only manifested through $E[S]$, with a smaller $E[S]$ leading to higher energy-efficiency. The impact of setup time on mean delay is manifested via $E[S]$ and $c_{s}^{2}$. When $E[S]$ is given, the mean delay is a monotonically increasing function of $c_{s}^{2}$. When $c_{s}^{2}$ is given, the mean delay is a monotonically increasing function of $E[S]$.

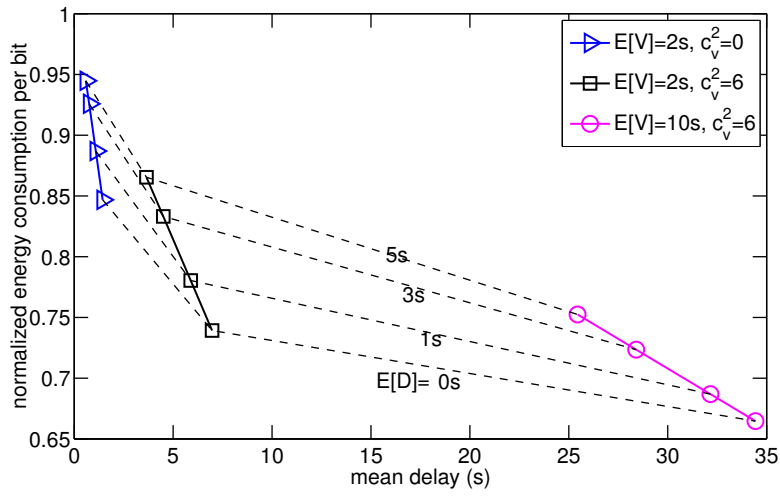

Fig. 4. For the MV policy, the energy consumption per bit (normalized by the case without sleep mode) vs. mean delay by changing close-down time for different vacation times with mean value $E[V]$ and coefficient of variation $c_{v}^{2}$ are shown. (for the case of exponentially distributed close-down time $D$ with different expected values, exponentially distributed setup time $S$ with $E[S]=0.5 \mathrm{~s}$ and $E_{\text {sniff }}=0$.)

3) Impact of $N$ : When (19) holds, a larger $N$ leads to higher energy-efficiency, and the mean delay is a monotonically increasing function of $N$ if and only if the following inequality holds

$$
\left(1-c_{s}^{2}\right) D^{*}(\lambda)+1 /(\lambda E[S])\left[2-3 D^{*}(\lambda)\right] \geq 0 .
$$

Otherwise, there exists an optimal value $N^{*}$ which can achieve the minimum mean delay, where

$$
\begin{aligned}
N^{*} & =-\left[\lambda E[S]+\frac{1-D^{*}(\lambda)}{D^{*}(\lambda)}\right] \\
& +\sqrt{\lambda^{2} c_{s}^{2} E[S]^{2}+\lambda E[S]+\left[\frac{1-D^{*}(\lambda)}{D^{*}(\lambda)}\right]^{2}} .
\end{aligned}
$$

For practical situations, $N$ can only be an positive integer. Hence, one should interpret (21) as saying that the minimum mean delay occurs between two integer values $\left\lfloor N^{*}\right\rfloor$ and $\left\lceil N^{*}\right\rceil$. A numerical result is shown in Fig. 5. In addition, when $c_{s}^{2} \leq 1$, the mean delay is a monotonically increasing function of $N$.

4) Impact of Close-down Time: When (19) holds, similar to the case of the SV and MV policies, by changing the closedown time, a linear tradeoff between energy-efficiency and mean delay exists since

$$
\begin{aligned}
\bar{P}_{\mathrm{v}}^{(\mathrm{N})}= & P_{0}-\frac{2 \lambda T_{\mathrm{v}}^{(\mathrm{N})}}{N^{2}-N+2 \lambda E[S] N+\lambda^{2} E\left[S^{2}\right]} \\
& \cdot\left[N\left(P_{0}-P_{\mathrm{SL}}-P_{\text {sniff }}\right)+\lambda E[S]\left(P_{\mathrm{CD}}-P_{\mathrm{ST}}\right)\right] .
\end{aligned}
$$

\section{Optimal EnERgy-Delay Tradeoff}

To achieve optimal energy-delay tradeoff, we attempt to design energy-optimal operation under a given mean delay constraint. The optimization problem is formulated as follows:

$$
\begin{array}{lll}
\mathrm{L1} 1: & \min & E_{\mathrm{bit}} \\
& \text { s.t. } & T \leq t_{0}
\end{array}
$$




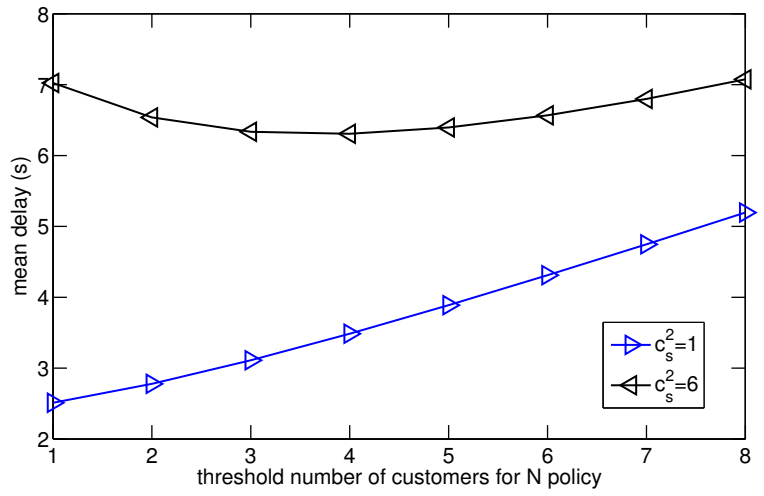

Fig. 5. For the $N$ policy, mean delay vs. threshold number of customers $(N)$ in different conditions are shown. (for the case of setup time with mean value $E[S]=3$ s and different coefficients of variation $c_{s}^{2}$ and exponentially distributed close-down time $D$ with $E[D]=1$ s. )

where $t_{0}$ denotes the mean delay constraint. We focus on the control of sleep mode operation, i.e., the choice of vacation time and hysteresis time (modeled as close-down time) in the $\mathrm{SV}$ and MV policies, the choice of $N$ and hysteresis time in the $N$ policy.

Since the impact of sleep mode operation on energyefficiency is only reflected in $\bar{P}_{\mathrm{v}}$, and the impact of sleep mode operation on mean delay is only reflected in $T_{\mathrm{v}}$, the problem L1 can be further simplified.

Here we use the SV policy as an example. Referring to the discussion in Section IV, we only consider the case when the vacation time is constant with value $v$, which means $c_{v}^{2}=0$ and $E[V]=v$. As a result, problem $\mathrm{L} 1$ is equivalent to the following problem.

$$
\begin{aligned}
& \text { L2: } \min _{v, D^{*}(\lambda)} \quad \bar{P}_{\mathrm{v}}^{(\mathrm{SV})} \\
& \text { s.t. } \quad T_{\mathrm{v}}^{(\mathrm{SV})} \leq t_{0}^{\prime} \\
& D^{*}(\lambda) \in(0,1]
\end{aligned}
$$

where $t_{0}^{\prime}$ denotes equivalent delay constraint, in terms of $t_{0}^{\prime}=$ $t_{0}-T_{M / G / 1}$. Recall that the impact of close-down time on both energy-efficiency and mean delay is only manifested through $D^{*}(\lambda)$.

We solve the problem L2 in the following two steps:

Step 1: As we mentioned before, when the condition for sleeping gain holds, $T_{\mathrm{v}}^{(\mathrm{SV})}$ is a monotonically increasing function of $D^{*}(\lambda)$, while on the other hand, $\bar{P}_{\mathrm{v}}^{(\mathrm{SV})}$ is a monotonically decreasing function of $D^{*}(\lambda)$. As a result, if we omit the constraint (24) in problem L2, for each $v, D^{*}(\lambda)$ which optimizes the energy-efficiency while satisfying the mean delay constraint is just the one satisfying $T_{\mathrm{v}}^{(\mathrm{SV})}=t_{0}^{\prime}$, which is

$$
D^{*}(\lambda)=\frac{2 t_{0}^{\prime}}{g_{1}(v)-2 t_{0}^{\prime} g_{2}(v)},
$$

where

$$
\begin{gathered}
g_{1}(v)=\lambda\left(v^{2}+2 v E[S]+E\left[S^{2}\right]\right), \\
g_{2}(v)=\lambda(v+E[S])+\mathrm{e}^{-\lambda v} S^{*}(\lambda)-1 .
\end{gathered}
$$

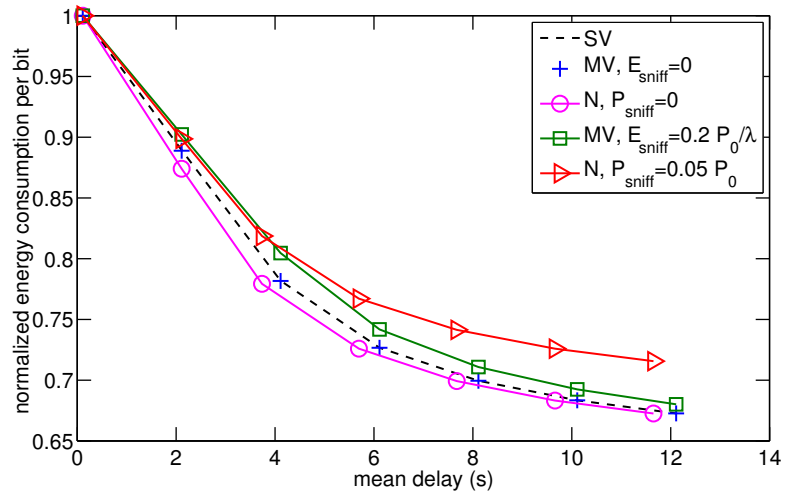

Fig. 6. Energy consumption per bit (normalized by the case without sleep mode) vs. mean delay for different wake-up policies with different sniffing costs are shown. (for the case of exponentially distributed setup time $S$ with $E[S]=1 \mathrm{~s}$. )

Then the problem L2 is equivalent to the following problem:

$$
\begin{array}{rll}
\text { L2.1: } & \min _{v} & \frac{2 t_{0}^{\prime}\left[v\left(P_{\mathrm{SL}}-P_{0}\right)+E[S]\left(P_{\mathrm{ST}}-P_{0}\right)\right]}{v^{2}+2 v E[S]+E\left[S^{2}\right]} \\
\text { s.t. } & v\left(P_{0}-P_{\mathrm{SL}}\right)>E[S]\left(P_{\mathrm{ST}}-P_{0}\right),
\end{array}
$$

where the constraint is the condition for sleeping gain. The optimum solution of L2.1 is

$$
v_{0}^{*}=\max \left\{v_{0}, E[S] \frac{P_{\mathrm{ST}}-P_{0}}{P_{0}-P_{\mathrm{SL}}}\right\},
$$

where

$$
\begin{aligned}
v_{0} & =\frac{1}{P_{0}-P_{\mathrm{SL}}}\left[E[S]\left(P_{\mathrm{ST}}-P_{0}\right)\right. \\
& \left.+\sqrt{E[S]^{2}\left(P_{\mathrm{ST}}-P_{0}\right) G_{3}^{(\mathrm{SV})}+E\left[S^{2}\right]\left(P_{0}-2 P_{\mathrm{SL}}\right)^{2}}\right],
\end{aligned}
$$

with

$$
G_{3}^{(\mathrm{SV})}=P_{\mathrm{ST}}+P_{0}-2 P_{\mathrm{SL}} .
$$

It should be noted that $v_{0}^{*}$ remains the same for different values of $t_{0}^{\prime}$, which can be easily understood since the objective function in L2.1 is a linear function of $t_{0}^{\prime}$.

As a result, when

$$
t_{0}^{\prime} \leq \frac{1}{2} \cdot \frac{g_{1}\left(v_{0}^{*}\right)}{1+g_{2}\left(v_{0}^{*}\right)},
$$

the optimum solution of problem L2 is

$$
v=v_{0}^{*}, \quad D^{*}(\lambda)=\frac{2 t_{0}^{\prime}}{g_{1}\left(v_{0}^{*}\right)-2 t_{0}^{\prime} g_{2}\left(v_{0}^{*}\right)} .
$$

Step 2: When (26) does not hold, the optimum solution of problem L2 is $D^{*}(\lambda)=1$, which means the length of hysteresis time is 0 , and the optimal vacation time $v$ is the solution of the following problem

$$
\begin{array}{ccc}
\text { L2.2: } \min _{v} & \frac{v\left(P_{\mathrm{SL}}-P_{0}\right)+E[S]\left(P_{\mathrm{ST}}-P_{0}\right)}{\mathrm{e}^{-\lambda v} S^{*}(\lambda)+\lambda(v+E[S])} \\
\text { s.t. } & v\left(P_{0}-P_{\mathrm{SL}}\right)>E[S]\left(P_{\mathrm{ST}}-P_{0}\right) \\
& \frac{\lambda\left(v^{2}+2 v E[S]+E\left[S^{2}\right]\right)}{\lambda(E[S]+v)+\mathrm{e}^{-\lambda v} S^{*}(\lambda)} \leq 2 t_{0}^{\prime} .
\end{array}
$$


When (13) holds, $\bar{P}_{\mathrm{v}}^{(\mathrm{SV})}$ is a monotonically decreasing function of $v$ while $T_{\mathrm{v}}^{(\mathrm{SV})}$ is monotonically increasing. In addition, a sufficient condition for (13) is $c_{s}^{2} \leq 1$. As a result, when $c_{s}^{2} \leq$ 1 , the solution of problem L2.2 is the one satisfying

$$
\frac{\lambda\left(v^{2}+2 v E[S]+E\left[S^{2}\right]\right)}{\lambda(E[S]+v)+\mathrm{e}^{-\lambda v} S^{*}(\lambda)}=2 t_{0}^{\prime}, v \geq \frac{P_{\mathrm{ST}}-P_{0}}{P_{0}-P_{\mathrm{SL}}} .
$$

Then the numerical result can be solved simply by a bisection method. Since in practical conditions, the coefficient of variance of setup time $\left(c_{s}^{2}\right)$ is relatively small, this method can be used in the most cases.

The two-step optimization method above is based on the linear tradeoff relationship between energy-efficiency and mean delay, obtained by changing close-down time. This can be explained as follows. Because of the linear relationship, the objective function in problem L2.1 is a linear function of the delay constraint. Hence, the optimal vacation time of L2.1 remains the same when mean delay constraint changes. As a result, the constraint (24) in problem L2 is equivalent to the inequality (26), leading to the optimization method above. For the MV and $N$ policies, since this linear tradeoff again exists, it can be solved in a similar way.

Fig. 6 shows the numerical results for the optimal energydelay relationships for different wake-up policies. We refer to [13] and [16] for the parameter values, and set $P_{0}=130 \mathrm{~W}$, $\Delta_{p}=4.7, P_{\mathrm{SL}}=75 \mathrm{~W}, \lambda=1 \mathrm{~s}^{-1}$ and $\bar{L}_{\text {data }}=40 \mathrm{Kbit}$. We further assumes that $\rho=0.1$ and $P_{\mathrm{ST}}=2 P_{0}$.

From the numerical results, when sniffing cost is not considered, both MV and $N$ policies have a better energy-delay relationship than the SV policy. However, these advantages diminish when the sniffing cost increases. For the case we study, when $E_{\text {sniff }} \geq 0.006 P_{0} / \lambda$, the $\mathrm{SV}$ policy is uniformly better than the MV policy, and when $P_{\text {sniff }} \geq 0.04 P_{0}$, the $\mathrm{SV}$ policy is uniformly better than the $N$ policy. In addition, there is a linear relationship between energy consumption per bit and mean delay for all the wake-up policies when the mean delay is relatively small. This is because when the mean delay constraint is relatively small, the optimal energydelay relationship is achieved by changing hysteresis time. Using the SV policy as an example, when (26) holds, the optimal vacation time remains the same, and the value of hysteresis time changes with the change of the mean delay constraint. As mentioned in Section IV, a linear energy-delay relationship exists by changing hysteresis time. As a result, the optimal energy-delay relationship is linear when the mean delay constraint $t_{0}^{\prime}$ is below the threshold value.

\section{CONCLUSION}

We have proposed three wake-up policies, the single vacation policy, the multiple vacation policy and the $N$ policy, together with a hysteresis time for BS sleep mode operation in different scenarios. By modeling the systems as M/G/1 vacation queues with setup and close-down times, we have determined the key performance measures of interest, energyefficiency and mean delay. One conclusion that can be drawn from our result is that equipment suppliers should make the setup time of BS as short as possible and simultaneously reduce the variance of the setup times in order to improve the energy-efficiency and reduce the mean delay. By exploiting the impact of operation parameters, we find that energy can be traded off for delay in BS sleep mode operation, with the tradeoffs depending on the BS control policies.

We have formulated constrained optimization problems to achieve the optimal energy-delay relationship and to determine the optimal parameter settings, and have proposed a two-step optimization method. We find that for all the policies, when the mean delay constraint is below a threshold value, the optimal tradeoff can be achieved by changing the hysteresis time, while the optimal value of vacation time or the threshold number of waiting customers for BS mode-changing operation remains the same. In addition, when the mean delay constraint is below the threshold value, the optimal energy-delay tradeoff is linear. From the numerical results, we observe that both the multiple vacation policy and the $N$ policy have better performance than the single vacation policy without sniffing cost, however, these advantages diminish when the sniffing cost increases.

\section{ACKNOWLEDGMENT}

The authors would like to thank Dr. Jian Wu for helpful discussions. This work is sponsored in part by the National Basic Research Program of China (2012CB316001), the Nature Science Foundation of China (61201191, 60925002, 61021001), and Hitachi Ltd.

\section{REFERENCES}

[1] B. Rengarajan, G. Rizzo, and M. Marsan, "Bounds on QoS-constrained energy savings in cellular access networks with sleep modes," in 23th International Teletraffic Congress (ITC), Sept. 2011, pp. 47 -54.

[2] S.-E. Elayoubi, L. Saker, and T. Chahed, "Optimal control for base station sleep mode in energy efficient radio access networks," in IEEE INFOCOM2011, April 2011, pp. 106 -110.

[3] J. Gong, S. Zhou, and Z. Niu, "A dynamic programming approach for base station sleeping in cellular networks," IEICE Trans., vol. 95-B, no. 2, pp. 551-562, 2012.

[4] E. Oh and B. Krishnamachari, "Energy savings through dynamic base station switching in cellular wireless access networks," in IEEE GLOBECOM, Dec. 2010, pp. $1-5$.

[5] R. Berry and R. Gallager, "Communication over fading channels with delay constraints," IEEE Information Theory, vol. 48, no. 5, pp. 1135 -1149 , May 2002.

[6] K. Son, H. Kim, Y. Yi, and B. Krishnamachari, "Base station operation and user association mechanisms for energy-delay tradeoffs in green cellular networks," IEEE Selected Areas in Commun., vol. 29, no. 8, pp. $1525-1536$, Sept. 2011.

[7] J. Wu, Y. Wu, S. Zhou, and Z. Niu, "Traffic-aware power adaptation and base station sleep control for energy-delay tradeoffs in green cellular networks," in IEEE GLOBECOM, Dec. 2012.

[8] Z. Niu, J. Zhang, X. Guo, and S. Zhou, "On energy-delay tradeoff in base station sleep mode operation," in 13th IEEE International Conference on Communication Systems (ICCS2012), Nov. 2012.

[9] Y. Sakai, Y. Takahashi, Y. Takahashi, and T. Hasegawa, "A composite queue with vacation/set-up/close-down times for svcc in ip over atm networks," J. of the Oper. Res. Soc. Japan, vol. 41, no. 1, March 1998.

[10] Z. Niu and Y. Takahashi, "A finite-capacity queue with exhaustive vacation/close-down/setup times and markovian arrival processes," Queueing Syst. Theory Appl. (QUESTA), vol. 31, no. 1/2, pp. 1-23, Jan. 1999.

[11] Y. Wu, T. Shu, Z. Niu, and J. Zheng, "A vacation model with setup and close-down times for transmitter buffer of arq schemes," in IEEE Proceedings on Personal, Indoor and Mobile Radio Communications, PIMRC, vol. 3, Sept. 2003, pp. $2054-2058$. 
[12] Z. Niu, T. Shu, and Y. Takahashi, "A vacation queue with setup and close-down times and batch markovian arrival processes," Performance Evaluation, vol. 54, no. 3, pp. 225 - 248, 2003.

[13] M. A. Imran and et, al, "Energy efficiency analysis of the reference systems, areas of improvements and target breakdown," EARTH, Tech. Rep., 2011.

[14] H. Takagi, Queueing analysis: a foundation of performance evaluation Volume 1: Vacation and Priority Systems. Elsevier Science, 1991.

[15] B. T. Doshi, "Queueing Systems with Vacations - A Survey," Queueing Systems - Theory and Applications, vol. 1, pp. 29-66, 1986.

[16] H. Holtkamp and et al., "Definition and parameterization of reference systems and scenarios," EARTH, Tech. Rep., 2011. 